\title{
Importancia de la biopsia de hueso en el diagnóstico de las enfermedades metabólicas óseas
}

\author{
Importance of bone biopsy in the diagnose on the bone metabolic diseases
}

\author{
Arquímedes Hidalgo ${ }^{1,2, a}$, Gustavo Cerrillo ${ }^{3, b}$, Oscar Ruiz ${ }^{1,4, c}$, Gerardo Ronceros ${ }^{1,3, \mathrm{~d}}$, Fausto Garmendia ${ }^{1, \mathrm{e}}$, Ángela Chuquihara ${ }^{1,2, \mathrm{f}}$, \\ Janet Villegas ${ }^{1,2, g}$
}

${ }^{1}$ Instituto de Investigaciones Clínicas, Facultad de Medicina, Universidad Nacional Mayor de San Marcos. Lima, Perú.

${ }^{2}$ Servicio de Reumatología, Hospital Nacional Dos de Mayo. Lima, Perú.

${ }^{3}$ Departamento de Patología Clínica, Hospital Nacional Dos de Mayo. Lima, Perú.

${ }^{4}$ Servicio de Hematología, Hospital Nacional Dos de Mayo. Lima, Perú.

a Médico cirujano, ORCID: https://orcid.org/0000-0002-8354-2889

${ }^{\mathrm{b}}$ Médico cirujano, ORCID: https://orcid.org/0000-0001-6209-0214

${ }^{c}$ Médico cirujano, ORCID: https://orcid.org/0000-0002-7178-9673

${ }^{d}$ Médico cirujano, ORCID: https://orcid.org/0000-0003-2117-2357

${ }^{e}$ Médico cirujano, ORCID: https://orcid.org/0000-0002-6513-8743

${ }^{\dagger}$ Médico cirujano, ORCID: https://orcid.org/0000-0003-0386-4632

${ }^{g}$ Médico cirujano, ORCID: https://orcid.org/0000-0002-7836-211

An Fac med. 2021;82(3):194-8. / DOI: https://doi.org/10.15381/anales.v82i3.21720

Correspondencia:

Fausto Garmendia Lorena

garmendiafausto@gmail.com

Recibido: 2 de junio 2021

Aprobado: 19 de agosto 2021

Publicación en línea: 30 de noviembre 2021

Conflictos de interés: Los autores declaran no tener conflictos de interés.

Fuente de financiamiento: Fundación Hipólito Unanue

\section{Contribuciones de autoría:}

AH y FG participaron en la concepción y diseño del estudio. AH obtuvo el financiamiento del estudio. $A H, O R, F G$, ACH y JV participaron en la recolección y obtención de resultados. Todos los autores participaron en el análisis e interpretación de datos, redacción del manuscrito, revisión crítica del manuscrito, y aprobación de su versión final.

Citar como: Hidalgo A, Cerrillo G, Ruiz O, Ronceros G, Garmendia F, Chuquihara A, et al. Importancia de la biopsia de hueso en el diagnóstico de las enfermedades metabólicas óseas. An Fac med. 2021;82(3):194-8. DOl: https://doi. org/10.15381/anales.v82i3.21720

\section{Resumen}

Objetivo. Evaluar la importancia de las biopsias óseas en el diagnóstico de las enfermedades óseas metabólicas, su relación con la densitometría ósea (DMO) y los biomarcadores. Métodos. Estudio transversal, descriptivo. Se efectuaron biopsias de la cresta pelviana a 38 personas, 36 mujeres (34 menopáusicas y 2 premenopáusicas) y 2 varones. DMO en columna vertebral, cadera y/o antebrazo por absorciometría dual de rayos $X$. Se determinó calcio, fósforo, osteocalcina, fosfatasa alcalina, vitamina $D$, parathormona, hemoglobina, transaminasas, proteinas totales y fracciones en sangre y en orina, N-telopéptido de enlaces de colágeno tipo I, calcio y fósforo por métodos convencionales. Resultados. La edad promedio de 34 mujeres postmenopáusicas fue $58 \pm 7,79$ y el tiempo de la menopausia $11,48 \pm 8,76$ años; la DMO promedio en columna, cadera y antebrazo fue de $-2,88 \pm 1,16,-2,13 \pm 0,82$, y $-5,14 \pm 1,76$, respectivamente; la biopsia demostró osteoporosis en 33 y enfermedad de Paget en 1. Dos varones de 48 y 63 años, con DMO en antebrazo de -3,2 y -4,9, y osteoporosis en la biopsia. Mujer de 34 años con DMO -3 y osteoporosis en biopsia; y una mujer de 29 años con DMO -3 y osteomalacia en biopsia. Hubo correlación negativa entre la edad y duración de la menopausia con la disminución de la DMO. Conclusiones. Hubo correlación entre la edad y tiempo de menopausia con la disminución de la DMO. Una DMO baja no siempre es osteoporosis, puede tratarse de otra enfermedad ósea metabólica como osteomalacia o enfermedad de Paget. La biopsia ósea es el procedimiento más confiable para el diagnóstico de estas enfermedades.

Palabras clave: Enfermedades Óseas Metabólicas; Biopsias; Densitometria; Biomarcadores (fuente: DeCS BIREME).

\section{Abstract}

Objective. To evaluate the importance of bone biopsy in the diagnose of the bone metabolic diseases, its relationship with bone densitometry (BMD) and biomarkers. Methods. In a descriptive, transversal study, 38 biopsies of pelvic bone were made, 36 in female (34 postmenopausal and 2 premenopausal) and 2 in male. All had BMD in spine, hip and/or forearm measured by dual x-ray absorptiometry. Calcium, phosphorus, parathormone, vitamin D, alkaline phosphatase, osteocalcin, hemoglobin, transaminases, total protein and fractions were measured in blood and N-telopeptide of collagen type I, calcium and phosphorus in urine by conventional methods. Results. The mean age of 34 menopausal women was $58 \pm 7,79$ and menopause time was $11,48 \pm 8,76$ years; mean BMD in spine, hip and forearm were $-2,88 \pm 1,16,-2,13 \pm 0,82, y-5,14 \pm$ 1,76 , respectively; bone biopsy demonstrated osteoporosis in 33 y Paget's disease in one. Male persons 48 and 63 years old had BMD in forearm $-3,2$ and $-4,9$, and osteoporosis in biopsy. A woman 34 years old had a BMD of -3 and osteoporosis en bone biopsy, a woman 29 years old with BMD -3 presented osteomalacia in biopsy. There was a negative correlatión between age and time of menopause with BMD decrease. Conclusions. A negative correlation between the age and time of menopause with BMD decrease was found. A low BMD not always is osteoporosis, it might be other bone metabolic disease like osteomalacia or Paget's disease. Bone biopsy is a more trustworthy procedure for the diagnose of these diseases.

Keywords: Bone Diseases, Metabolic; Biopsy; Densitometry; Biomarkers (source: MeSH NLM) 


\section{INTRODUCCIÓN}

En la fisiología humana, el sistema esquelético cumple funciones muy importantes, tales como de sostén y de los movimientos corporales, de protección de órganos muy importantes como el cerebro, tórax, médula espinal y abdomen, participa en la hematopoyesis y se encuentra en un intercambio permanente muy activo de remodelación mediante la formación y resorción para mantener la estructura ósea estable ${ }^{(1)}$.

Las enfermedades metabólicas de hueso son alteraciones sistémicas del sistema óseo vinculadas a disturbios en el complejo sistema de su regulación humoral ${ }^{(2)}$; las más importantes y frecuentes son la osteoporosis, osteomalacia, raquitismo y la osteítis fibroquística del hiperparatiroidismo, y con menor frecuencia la enfermedad de Paget y la osteopetrosis. En la osteoporosis se produce una disminución de la masa ósea, deterioro de la microarquitectura del hueso y fragilidad que lo hace más susceptible para la producción de fracturas patológicas ${ }^{(3)}$. En el raquitismo y la osteomalacia existe una deficiente mineralización de la matriz proteica recientemente formada u osteoide, en el niño se denomina raquitismo, en el que al no haberse completado el proceso de formación del hueso se presentan deformaciones en el eje de los huesos; en cambio en la osteomalacia (osteo=hueso, malacia=blando) es la misma enfermedad, pero en adultos, sin que se presenten deformaciones muy evidentes de los huesos ${ }^{(4)}$. La osteítis fibroquística es la alteración de la arquitectura ósea más característica del hiperparatiroidismo ${ }^{(5)}$. La enfermedad de Paget es una enfermedad crónica del hueso caracterizada por una disarmonía entre la resorción y formación del hueso que puede comprometer a uno o varios huesos ${ }^{(6)}$.

En el presente estudio, se puntualiza la importancia que tiene la biopsia de hueso en el diagnóstico y diagnóstico diferencial de las enfermedades metabólicas óseas, así como las correlaciones que pudieran existir con la densitometría ósea y los marcadores bioquímicos involucrados en el metabolismo óseo.

\section{MÉTODOS}

\section{Tipo de estudio}

Se realizó un estudio observacional descriptivo, transversal, analítico.

\section{Población de estudio}

Participaron del estudio 38 personas, 36 mujeres y 2 varones, a quienes se efectuó $\mathrm{DMO}$, medidas en la columna (DCol), cadera (DCad) y/o antebrazo (Dant), efectuadas mediante absorciometría dual de rayos $X$ y evaluadas de acuerdo a los criterios de la $\mathrm{OMS}^{(7)}$ y la International Society for Clinical Densitometry (ISCD) ${ }^{(8)}$, que se expresó tanto en $\mathrm{g} / \mathrm{cm}^{2}$, así como en el score $\mathrm{T}$ y el score Z. La DMO se consideró normal cuando el score $T$ fue $\geq$ de-1,0/DS en columna, cadera y/o antebrazo; osteopenia cuando el score estuvo entre-1,0/DS y-2,5/DS y osteoporosis cuando fue inferior a-2,50/DS.

\section{Criterios de inclusión}

Pacientes atendidos en el Servicio de Reumatología del Hospital Dos de Mayo en el periodo marzo 2013 a marzo 2015, que presentaron una densitometría $\leq-2,5$ en forma no aleatorizada, y una vez que firmaron el consentimiento informado, tanto para el ingreso al estudio como para la biopsia de hueso.

\section{Criterios de exclusión}

Fueron los antecedentes patológicos de personas con osteoporosis secundaria (menopausia precoz, hipogonadismo, uso prolongado de corticoides, uso de anticonceptivos progestágenos de acción prolongada, sedentarismo, hiperprolactinemia, diabetes mellitus, enfermedades malignas tanto mielo como linfo-proliferativas, mieloma múltiple, mastocitosis sistémica, hipertiroidismo, anorexia nervosa, quimioterapia por cáncer de mama y cáncer de próstata) o que hubiesen usado terapia antiosteoporótica (bisfosfonatos, denosumab, moduladores selectivos de receptores estrogénicos, calcitonina, terapia de remplazo hormonal, calcio o vitamina D sustitutiva, etc).

\section{Biopsias óseas y marcadores bioquímicos}

Las biopsias óseas de la cresta iliaca fueron fijadas con formaldehido al $10 \%$, descalcificadas con ácido nítrico al 5\%, teñidas con coloración de hematoxilina- eosina y analizadas en un microscopio óptico por un médico especialista anatomopatólogo. Se determinó en sangre calcio, fósforo, osteocalcina, fosfatasa alcalina, 25-hidroxi-vitamina D $(25-\mathrm{OH}-$ D), parathormona, hemoglobina, transaminasas, proteínas totales y fracciones y en orina, N-telopéptido de enlaces de colágeno tipo I (NTX), calcio y fósforo por métodos convencionales.

\section{Análisis estadístico}

Se utilizó el programa SPSS.v23 para el análisis estadístico de la determinación de los promedios, desviación estándar, diferencia de los promedios y correlaciones entre los diferentes ítems estudiados. Los valores numéricos se expresaron como promedio \pm 1 desviación estándar y en la comparación de las diferencias fueron significativas cuando el valor $p$ fue menor de 0,05.

\section{Aspectos éticos}

El estudio fue aprobado por Comité de Ética del Hospital Nacional Dos de Mayo, Lima, Perú.

\section{RESULTADOS}

De las mujeres, 34 eran posmenopáusicas con una edad de $58 \pm 7,79$, tiempo de menopausia de 11,48 $\pm 8,76$ años y 2 mujeres premenopáusicas de 29 y 34 años; los 2 varones tuvieron 49 y 63 años. El diagnóstico histopatológico de las mujeres posmenopáusicas fue osteoporosis en 33 (Tabla 1 y Figura 1) y enfermedad de Paget en una; la mujer premenopáusica de 34 años con DCol-3,4 mostró osteoporosis en la biopsia y la de 29 años con Dcol-2,3, DCad-3 y DAnt-4,2 presentó osteomalacia en la biopsia. Los 2 varones de 48 y 63 años, con DAnt-3,2 y-4,9 respectivamente, tuvieron el diagnostico de osteoporosis en la biopsia (Tabla 2).

La DCol mostró una correlación negativa con la edad, menopausia, y con la fosfatasa alcalina. La Dcad tuvo una correlación negativa con la edad, positiva con Dcol y Dant. La Dant presentó una correlación negativa con la edad y la menopausia; y correlación positiva con la Dcad. Los marcadores bioquímicos de las mujeres menopáusicas no mostraron correlaciones. El 56,6\% de las mujeres con 
Tabla 1. Resultados de la DMO y biopsia ósea de 33 mujeres posmenopáusicas

\begin{tabular}{ccccccccc} 
Edad & $N^{* *}$ & Sexo & Meno & DMO Col & DMO Cad & DMO Ant & Biopsia Osea \\
\hline $44-49$ & 5 & $F$ & $5,6 \pm 5,52$ & $-1,82 \pm 1,30$ & $-1,54 \pm 0,24$ & $-3,88 \pm 0,60$ & Osteoporosis \\
\hline $52-59$ & 15 & $F$ & $6,6 \pm 5,33$ & $-2,95 \pm 0,91$ & $-2,10 \pm 0,75$ & $-4,99 \pm 1,39$ & Osteoporosis \\
\hline $60-79$ & 13 & $F$ & $19,4 \pm 7,11$ & $-3,26 \pm 1,16$ & $-2,46 \pm 0,94$ & $-5,78 \pm 2,17$ & Osteoporosis \\
\hline
\end{tabular}

*número de casos; Meno: duración de la menopausia en años; DMO: densitometría: Col: columna; Cad: cadera; Ant: antebrazo

Tabla 2. Resultados de las DMO y biopsia ósea de 5 pacientes

\begin{tabular}{|c|c|c|c|c|c|c|c|}
\hline Paciente & Sexo & edad & Meno & DMO Col & DMO Cad & DMO Ant & Biopsia Ósea \\
\hline 1 & M & 63 & - & $-1,1$ & & $-4,9$ & Osteoporosis \\
\hline 2 & M & 48 & - & & & $-3,3$ & Osteoporosis \\
\hline 3 & $\mathrm{~F}$ & 34 & - & -3 & & & Osteoporosis \\
\hline 4 & $\mathrm{~F}$ & 29 & - & $-2,3$ & -3 & -6 & Osteomalacia \\
\hline 5 & $\mathrm{~F}$ & 64 & 11 & -0.7 & & $-3,9$ & Enf. de Paget \\
\hline
\end{tabular}

Tabla 3. Marcadores bioquímicos de 33 mujeres menopáusicas

\begin{tabular}{lccccccccccccccc} 
Cas & Ps & PTH & VitD & Osteo & FA & Cr & PT & ALB & GLOB & TGO & TGP & Hb & NTX & Cau & Pu \\
9,17* & 4,30 & 23,5 & 29,6 & 32,4 & 127,4 & 0,72 & 6,84 & 4,18 & 2,70 & 28,3 & 39,0 & 12,3 & 86,4 & 142,3 & 0,93 \\
\hline $0,49^{* *}$ & 0,58 & 7,33 & 8,11 & 12,3 & 41,0 & 0,21 & 0,71 & 0,27 & 0,55 & 13,0 & 19,1 & 0,95 & 13,4 & 69,7 & 0,28 \\
\hline
\end{tabular}

*: promedio; **: desviación estándar; Cas: calcio sérico; Ps. Fósforo sérico; PTH: parathormona; VitD (25-hidroxi-D); Osteo: osteocalcina; FA: fosfatasa alcalina; Cr: creatinina en sangre; PT: Proteínas totales; ALB: albúmina; GLOB: globulinas; TGO: transaminasa glutámico oxalacética; TGP: transaminasa glutámico pirúvica; Hb: hemoglobina; NTX: N-telopéptido de enlaces de colágeno tipo I; Cau: calcio urinario; Pu: fósforo urinario.

osteoporosis tuvieron cifras bajas de vitamina $\mathrm{D}$, medida como $25-\mathrm{OH}-\mathrm{D}$, que tuvo una correlación con la concentración del calcio urinario (Tabla 3).
La paciente con diagnóstico histopatológico de enfermedad de Paget tuvo cifras altas de fosfatasa alcalina y NTX; y bajas de $25-0 \mathrm{H}-\mathrm{D}$ y hemoglobina. El pa-

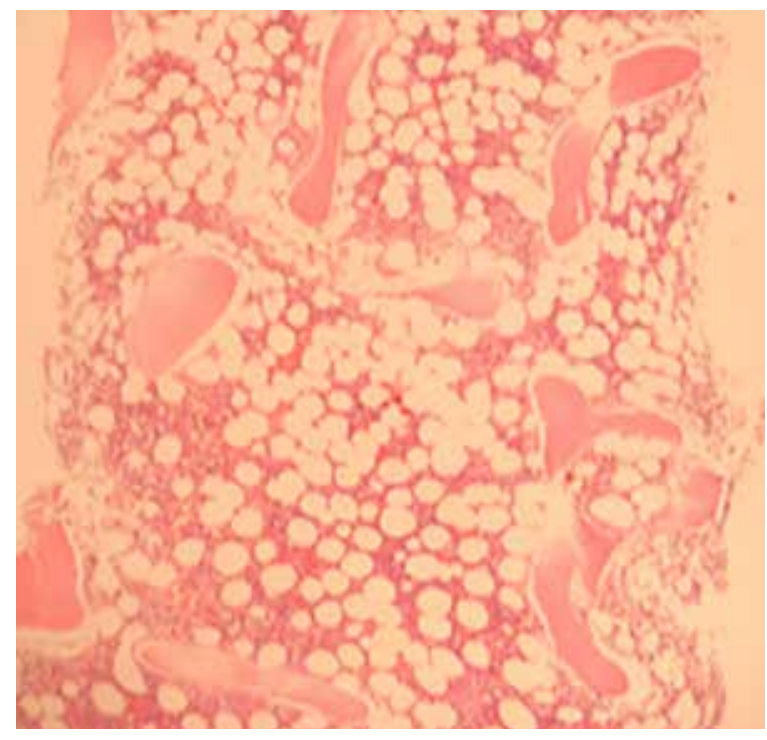

Figura 1. Osteoporosis ciente varón de 63 años con diagnóstico de osteoporosis solo tuvo una disminución en la hemoglobina; el paciente varón de 48 con diagnóstico de osteoporosis presentó cifras elevadas de osteocalcina y NTX y una ligera anemia. La paciente de 22 años con diagnóstico histopatológico de osteomalacia presentó calcio sérico iónico en el rango normal bajo, cifras elevadas de fosfatasa alcalina y de NTX. La paciente de 34 años con diagnóstico histopatológico de osteoporosis tuvo cifras elevadas de fosfatasa alcalina y NTX.

\section{DISCUSIÓN}

En el presente estudio se muestran los resultados de 38 biopsias de hueso en pacientes que tenían previamente determinaciones de la DMO en el rango de osteoporosis con el objeto de confirmar el diagnóstico definitivo de la enfermedad metabólica de hueso. En 33 mujeres posmenopáusicas y una premenopáusica, así 


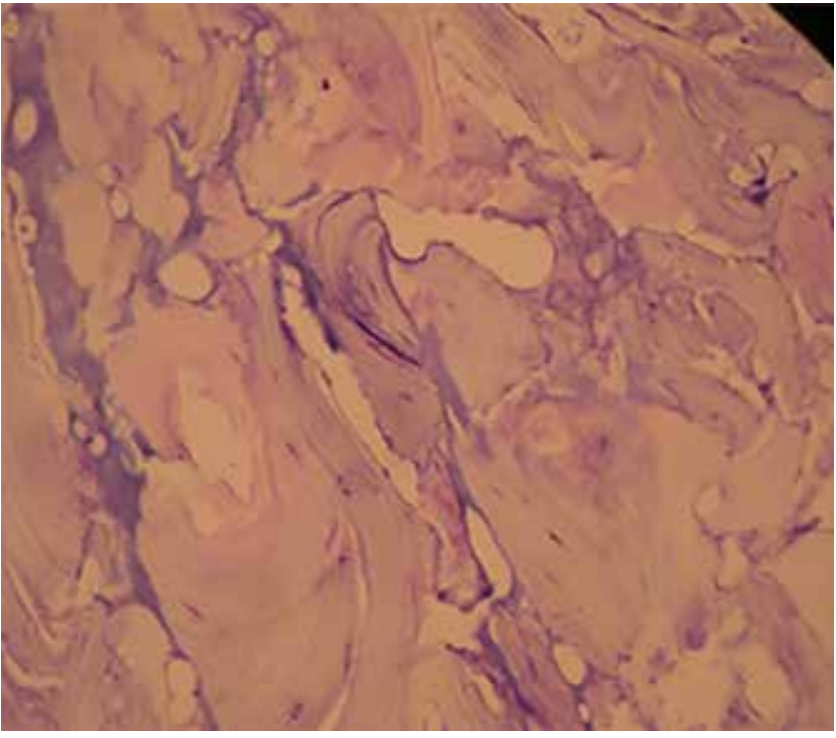

Figura 2. Osteomalacia

como en 2 varones, las biopsias de hueso confirmaron el diagnóstico de osteoporosis; en una mujer posmenopáusica con DMO baja, la biopsia reveló enfermedad de Paget y en un mujer premenopáusica la biopsia mostró osteomalacia; estos dos últimos resultados demuestran que una densitometría baja no siempre significa la existencia de osteoporosis y confiere a la biopsia de hueso un valor definitorio en el diagnóstico de estas enfermedades, tal como se puede ver en las imágenes de las biopsia de hueso mostradas ${ }^{(9,10)}$.
En la revisión de los repositorios bibliográficos PubMed, Scielo, Scopus no hemos encontrado referencias bibliográficas relacionadas a la biopsia de hueso para el diagnóstico y manejo de las enfermedades metabólicas de hueso en el Perú, por lo que consideramos que esta sería la primera publicación sobre este tema en el país.

La osteoporosis es la enfermedad ósea metabólica más frecuente, clínicamente silente hasta que se complica con fracturas patológicas de la columna vertebral, cadera y/o de la extremidad distal del antebrazo ${ }^{(3)}$

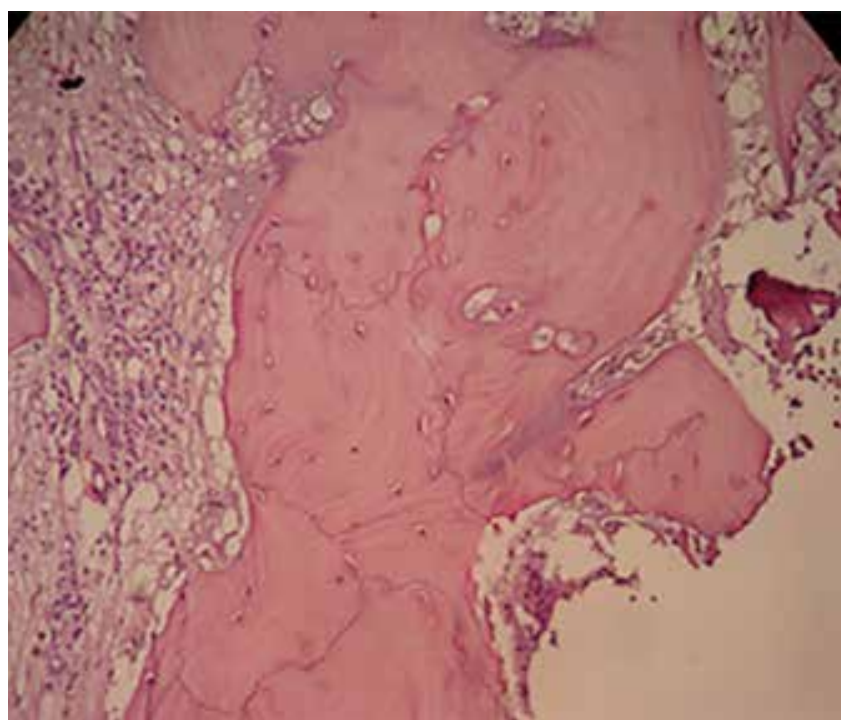

Figura 3. Enfermedad de Paget

esto se corrobora en nuestros hallazgos, en el que la mayoría de pacientes (33 mujeres y 2 varones) presentaron una osteoporosis primaria, derivada de los fenómenos biológicos naturales como la menopausia (tipo I) (11) y del proceso natural de envejecimiento (tipo II) ${ }^{(12,13)}$, y se ha descartado a las osteoporosis secundaria, que subyace a diversos procesos patológicos o el uso de medicamentos ${ }^{(14,15)}$. La osteoporosis es más frecuentemente en mujeres, pero como se ha mostrado en este estudio, se debe tener presente su existencia en varones ${ }^{(16,17,18)}$. Existe relación con la edad y el tiempo de menopausia; sin embargo, también se puede presentar en adultos jóvenes ${ }^{(19,20,21)}$. Los biomarcadores del metabolismo óseo se encontraron dentro de límites normales en la osteoporosis y no ayudaron a diferenciarla de las otras enfermedades metabólicas de hueso ${ }^{(22)}$

En la osteomalacia se presentan alteraciones del metabolismo del calcio y/o la vitamina D por muy diversas causas como deficiente ingesta, mala absorción, hipofosfatemia, hipofosfatasia, pérdida urinaria incrementada de calcio, acidosis tubular renal, osteomalacia oncógena (23-26).

En una paciente, la biopsia de hueso permitió efectuar el diagnóstico de la enfermedad de Paget, condición metabólica crónica del hueso caracterizada por una alteración del remodelado óseo que compromete en diversa extensión el sistema esquelético ${ }^{(6,27,28)}$.

Entre las limitaciones de nuestra investigación se encuentra el bajo número de casos de varones y de mujeres premenopáusicas que no permiten extrapolar los hallazgos, así como la no aleatorización de los participantes del estudio.

Concluimos que la biopsia de hueso se encuentra indicada para el diagnóstico diferencial de las enfermedades metabólicas de hueso, para dirimir los diagnósticos en personas que tienen densitometrías bajas y sintomatología que advierta que no se trata de una osteoporosis (29), en los casos de falta de respuesta al tratamiento de la osteoporosis (30) desde que esta enfermedad suele transcurrir sin molestias a no ser que se produzcan fracturas patológicas, así como también cuando tengan una DMO baja en una edad que no se suele observar. La biopsia de hueso en la osteoporosis se ha utilizado también 2 veces por persona para evaluar la respuesta al tratamiento ${ }^{(31)}$. 
La densitometría ósea es un procedimiento valioso para la detección de enfermedades metabólicas óseas, pero no permite efectuar el diagnóstico diferencial definitivo con las otras enfermedades de esta naturaleza, desde que ella puede estar baja en la osteoporosis, osteomalacia, osteítis fibroquística del hiperparatiroidismo y la enfermedad de Payet.

Los marcadores bioquímicos del metabolismo óseo son una ayuda complementaria para el diagnóstico, pero no son útiles para el diagnóstico diferencial.

\section{REFERENCIAS BIBLIOGRÁFICAS}

1. Garmendia F. Metabolismo normal y patológico de hueso. Diagnóstico 2011; 50 (1): 11-13.

2. Albright $F$, Reifenstein EC Jr. The parathyroid gland and metabolic bone disease. Baltimore, MD: The Williams and Wilkins Company; 1948.

3. Lewiecki EM, Bilezikian JP, Giangregorio L, Greenspan SL, Khosla S, Kostenuik P et al. Proceedings of the 2018 Santa Fe Bone Symposium: Advances in the Management of Osteoporosis. J Clin Densitometry. 2019; 22, (1): 1-19. DOI: https://doi.org/10.1016/j.jocd.2018.09.010

4. Elbossaty WF. Mineralization of Bones in Osteoporosis and Osteomalacia. Ann Clin Lab Res. 2017; 5 (4): 201-4. DOI: 10.21767/2386-5180.1000201

5. Jawaid I, Rajesh S. Hyperparathyroidism (primary) NICE guideline: diagnosis, assessment, and initial management. $\mathrm{Br} J$ Gen Pract. 2020 25;70(696):362-363. DOI: 10.3399/bjgp20X710717

6. Hsu E. Paget's disease of bone: updates for clinicians. Curr Opin Endocrinol Diabetes Obes. 2019; 26(6): 329-334. DOI: 10.1097/ MED.0000000000000503

7. World Health Organization [Internet]. Assesment of fracture risk and its application to screening for postmenopausal osteoporosis: report of a WHO study group [Fecha de acceso: 8 de marzo 2020]. Disponible en: https://apps.who.int/iris/ handle/10665/39142 10.

8. International Society for Clinical Densitometry [Internet]. Official Position Adults, 2019 [Fecha de acceso: 8 de marzo 2020]. Disponible en: https:// www.iscd.org/official-

9. Narla RR, Ott SM. Structural and metabolic assessment of bone. Handb Exp Pharmacol. 2020; 262:369-396. DOI: 10.1007/164_2020_376. PMID: 32885312

10. Malluche $\mathrm{HH}$, Mawad $\mathrm{H}$, Monier-Faugere MC. Bone biopsy in patients with osteoporosis. Curr Osteoporos Rep. 2007; 5(4):146-52. DOI: 10.1007/ s11914-007-0009-x

11. Hidalgo A, Ronceros G, Cerrillo G, Ruiz A, Garmendia F, Villegas J, Chuquihuara A. Características del metabolismo óseo en mujeres con osteoporosis posmenopáusica de un hospital de Lima. An Fac med. 2019; 80(3):283-7. DOI: https://10.15381/ anales.803.16845

12. Qadir A, Liang S, Wu Z, Chen Z, Hu L, Qian A. Senile Osteoporosis: The involvement of differentiation and senescence of bone marrow stromal cells. Int J Mol Sci. 2020; 21(1):349. DOI: 10.3390/ ijms21010349

13. Coughlan T, Dockery F. Osteoporosis and fracture risk in older people. Clin Med (Lond). 2014;14(2):187-191. DOI: 10.7861/clinmedicine.14-2-187

14. Mirza F, Canalis E. Secondary osteoporosis: pathophysiology and management. Eur J Endocrinol. 2015; 173 (3): 131-RR151. DOI: 10.1530/ EJE-15-0118

15. Colangelo L, Biamonte F, Pepe J, Cipriani C, Minisola S. Understanding and managing secondary osteoporosis. Expert Rev Endocrinol Metab. 2019; 14(2): 111-122. DOI: 10.1080/17446651.2019.1575727

16. Garmendia-Lorena F, Pando-Álvarez R, RuizFranco O, Salas-Pérez M, Chuquihuara-Rodríguez A, Hidalgo-Garcia A y col. Densidad de la masa ósea y marcadores bioquímicos del metabolismo óseo: estudio transversal en adultos residentes de Lima Metropolitana, Perú. An Fac med. 2020; 81(2):148-53. DOI: https://doi. org/10.15381/ anales.v81i2.18106

17. Kanis JA, Melton LJ, Christiansen C, Johnston CC, Khaltaev N. Perspective. The Diagnosis of Osteoporosis. J Bone Mineral Research. 1994; 9 (8): 1137-41. DOI: 10.1002/jbmr.5650090802

18. Haney EM, Bliziotes M. Male Osteoporosis: new insights in an understudied disease. Curr Opin Rheumatol. 2008; 20(4): 423-428. DOI: 10.1097/ BOR.0b013e3283025eb0

19. Barrios-Moyano A, De la Peña-García C. Prevalencia de osteoporosis y osteopenia en pacientes laboralmente activos. Acta Ortop Mex. 2018;32(3):131-133
20. McLendon AN, C Woodis CB. A review of osteoporosis management in younger premenopausal women. Women's Health. 2014; 10 (1): 59-77. DOI: 10.2217/whe.13.73

21. Cohen A. Premenopausal osteoporosis. Endocrinol Metab Clin N Am. 2017; 46 (1): 117-133. DOI:10.1016/j.ecl.2016.09.007

22. Greenblatt MB, Tsai JN, Wein MN. Bone turnover markers in the diagnosis and monitoring of metabolic bone disease. Clin Chem. 2017;63(2):464-474 DOI: 10.1373/clinchem.2016.259085

23. Figueroa R, Melgar E, Jo N, Garcia O. Intestinal lactase deficiency in an apparently normal Peruvian population. Am J Digest Dis. 1971:16 (10): 881-889.

24. Felicita Mendoza JF, Moreno MA, León JV, Patiño MA, Bejarno FX. Osteomalacia. Diagnóstico y tratamiento. Revista Cubana Reumatología. 2019; 21(2): 1-11.

25. Elbossaty WF. Mineralization of bones in osteoporosis and osteomalacia. Ann Clin Lab Res. 2017 5(4): 201-204. DOI: 10.21767/2386-5180.1000201

26. Dey B, Gochhait D, Subramanian H, Ponnusamy M. Oncogenic Osteomalacia: An Approach to Diagnosis with a Case Report. J Clin Diagn Res. 2017; 11(4):ED05-ED07. DOI: 10.7860/ JCDR/2017/25055.9634

27. Carrillo-Ñáñez L, Atencia-Matute F, Ortiz-Zegarra C, Hernández-López M. Enfermedad de Paget ósea craneal con hidrocefalia obstructiva y tuberculosis miliar. Rev Soc Peru Med Interna. 2011; 24 (1): 46-50.

28. Ralston SH, Corral-Gudino L, Cooper C, Francis RM, Fraser WD, Gennari L, et al. Diagnosis and Management of Paget's Disease of Bone in Adults: A Clinical Guideline. J Bone Miner Res. 2019; 34(4): 579-604. DOI: 10.1002/jbmr.3657

29. Fardellone P. Osteoporosis diagnosis and screening (Diagnostic et despistage de l'ostéoporose). La Revue du Practicien, abstract. 2020; 70 (9): 1023-1026

30. Léger $\mathrm{B}$, Fardellone $\mathrm{P}$, Cormier $\mathrm{C}$, Ostertag $\mathrm{A}$, Funck-Brentano T, Fabre $S$, et al. Inadequate response to treatment reveals persistent osteoclast bone resorption in osteoporotic patients. Bone. 2021; 153. DOI. 10.1016/j.bone.2021.116167

31. Falgayrac G, Farlay D, Poncon C, Béhal H, Gardegaront $\mathrm{M}$, Ammann $\mathrm{P}$, et al. Bone matrix quality in paired iliac bone biopsies from postmenopausal women treat for 12 months with strontion ranelate or alendronate. Bone. 2021; 153. DOI: 10.1016/j. bone. 2021.116107. 